\title{
TORSIONAL RIGIDITY OF SUBMANIFOLDS WITH CONTROLLED GEOMETRY
}

\author{
A. HURTADO ${ }^{\natural}$, S. MARKVORSEN\#, AND V. PALMER*
}

\begin{abstract}
We prove explicit upper and lower bounds for the torsional rigidity of extrinsic domains of submanifolds $P^{m}$ with controlled radial mean curvature in ambient Riemannian manifolds $N^{n}$ with a pole $p$ and with sectional curvatures bounded from above and from below, respectively. These bounds are given in terms of the torsional rigidities of corresponding Schwarz symmetrizations of the domains in warped product model spaces. Our main results are obtained using methods from previously established isoperimetric inequalities, as found in e.g. [MP4] and [MP5]. As in [MP4] we also characterize the geometry of those situations in which the bounds for the torsional rigidity are actually attained and study the behavior at infinity of the so-called geometric average of the mean exit time for Brownian motion.
\end{abstract}

\section{INTRODUCTION}

Given a precompact domain $D$ in a complete Riemannian manifold $\left(M^{n}, g\right)$, the torsional rigidity of $D$ is defined as the integral

$$
\mathcal{A}_{1}(D)=\int_{D} E(x) d \sigma,
$$

where $E$ is the smooth solution of the Dirichlet-Poisson equation

$$
\begin{aligned}
\Delta^{M} E+1 & =0 \text { on } D \\
\left.E\right|_{\partial D} & =0
\end{aligned}
$$

2000 Mathematics Subject Classification. Primary 53C42, 58J65, 35J25, $60 \mathrm{~J} 65$.

Key words and phrases. Riemannian submanifolds, extrinsic balls, torsional rigidity, mean exit time, isoperimetric inequalities, Faber-Krahn inequalities, Schwarz symmetrization.

\# Supported by the Danish Natural Science Research Council and the Spanish MECDGI grant MTM2007-62344.

* Supported by Spanish MEC-DGI grant No.MTM2007-62344, the Caixa Castelló Foundation and a grant of the Spanish MEC Programa de Estancias de profesores e investigadores españoles en centros de enseñanza superior e investigación extranjeros.

${ }^{\natural}$ Supported by Spanish MEC-DGI grant No.MTM2007-62344, the Caixa Castelló Foundation. 
Here $\Delta^{M}$ denotes the Laplace-Beltrami operator on $\left(M^{n}, g\right)$. The function $E(x)$ represents the mean time of first exit from $D$ for a Brownian particle starting at the point $x$ in $D$, see [Dy].

The name torsional rigidity of $D$ stems from the fact that if $D \subseteq \mathbb{R}^{2}$, then $\mathcal{A}_{1}(D)$ represents the torque required per unit angle of twist and per unit length when twisting an elastic beam of uniform cross section $D$, see [Ba] and [PS]. As in [MP4] we consider a Saint-Venant type problem, namely, how to optimize the torsional rigidity among all the domains having the same given volume in a given space or in some otherwise fixed geometrical setting. Here we restrict ourselves to a particular class of subsets, namely the extrinsic balls $D_{R}$ of a submanifold $P$ properly immersed with controlled mean curvature into an ambient manifold with suitably bounded sectional curvatures.

The proof of the Saint-Venant conjecture in the general context of Riemannian geometry makes use of the concept of Schwarz-symmetrization and like the Rayleigh conjecture concerning the fundamental tone it also hinges upon the proof of the Faber-Krahn inequality, which in turn is based on isoperimetric inequalities satisfied by the domains in question, (see $[\mathrm{Mc}]$ ).

Under extrinsic curvature restrictions on the submanifold and intrinsic curvature restrictions on the ambient manifold we show in Theorem 3.2 that the extrinsic balls satisfy strong isoperimetric inequalities, specifically lower and upper bounds for the $\infty$-isoperimetric quotient $\operatorname{Vol}\left(\partial D_{R}\right) / \operatorname{Vol}\left(D_{R}\right)$, where the bounds are given by corresponding $\infty$-isoperimetric quotients of certain geodesic balls in tailor-made warped product spaces.

As in [Pa2], [Ma1], and [MP4], the comparison is obtained essentially by transplanting the radial solution of a Poisson equation defined in the radially symmetric model space from that model to the extrinsic $R$-balls $D_{R}$ in the submanifold $P$.

Once we have this isoperimetric information at hand, we then apply it to get bounds for the torsional rigidity of the extrinsic balls. One key result on the way to upper and lower bounds for the torsional rigidity is Theorem 4.4, which shows a fundamental equality between the integral of the transplanted radial solution of the Poisson equation in $D_{R}$ and the corresponding integral of its Schwarz-symmetrization in the model space.

As a consequence of the isoperimetric inequalities in Theorem 3.2 and the Schwarz symmetrization identity in Theorem 4.4, we obtain lower and upper bounds for the torsional rigidity of the extrinsic balls in submanifolds with 
controlled mean curvature in ambient manifolds with radial sectional curvatures bounded from below (Theorem 5.1) or from above (Theorem 5.3), respectively. Upper bounds for the torsional rigidity of such domains were found in [MP4] for the special cases where the submanifold is minimal.

In the work $[\mathrm{BBC}]$ the existence of regions in $\mathbb{R}^{m}$ with finite torsional rigidity and yet infinite volume were considered. To get to such regions, the authors assume Hardy inequalities for these domains. The geometric effect of this assumption is to make the volume of the boundary of the regions relatively large in comparison with the enclosed volume. In consequence, the Brownian diffusion process finds sufficient outlet-volume to escape at the boundary, giving in consequence a small mean exit time and at the same time a small incomplete integral of the mean exit time, i.e. a bounded torsional rigidity.

Inspired by this result, it was initiated in [MP4] the study of the behaviour at infinity of the geometric average of the mean exit time for Brownian motion. Specifically, given the quotient $\mathcal{A}_{1}\left(D_{R}\right) / \operatorname{Vol}\left(D_{R}\right)$, we may consider the limit of this quotient for $R \rightarrow \infty$ as a measure of the volume-relative swiftness (at infinity) of the Brownian motion defined on the entire submanifold. In this paper, we establish a set of curvature restrictions that guarantee the finiteness of the average mean exit time at infinity, meaning that the Brownian diffusion process is moving relatively fast to infinity (see Corollary 7.3), and a dual version of this result, i.e. a set of curvature restrictions which guarantee in turn that the diffusion is moving relatively slow to infinity (see Corollary 7.2).

Concerning these last results, we should remark that it was proved in [MP4] that this quotient is unbounded for geodesic balls in all Euclidean spaces as $R \longrightarrow \infty$, while it is bounded for geodesic balls in simply connected space-forms of constant negative curvature. Therefore, transience is not in itself sufficient to give finiteness of the geometric average of the mean exit time, as is exemplified by $\mathbb{R}^{n}$ for all $n \geq 3$. We refer to [MP2, MP3, MP5] for results concerning general transience conditions for submanifolds.

Outline of the paper. Section 2 is devoted to the precise definitions of extrinsic balls, the warped product spaces that we use as models and to the description of the general set-up of our comparison analysis: the comparison constellations. In sections 3 and 4 we formulate the isoperimetric inequalities and the integral equalities for the Schwarz-symmetrization of the solution of the Poisson equation, respectively. The main comparison results for the Torsional Rigidity are stated and proved in Section 5, and finally, in sections 
6 and 7 we present an intrinsic analysis of these results and consider the behavior of the averaged mean exit time at infinity, respectively.

Acknowledgements. This work has been partially done during the stay of the third named author at the Department of Mathematics at the Technical University of Denmark and at the Max Planck Institut für Mathematik in Bonn, where he enjoyed part of a sabbatical leave, funded by a grant of the Spanish Ministerio de Educación y Ciencia. He would like to thank these institutions for their support during this period and to thank the staff of the Mathematics Department at DTU and the MPIM for their cordial hospitality.

\section{Preliminaries and Comparison Setting}

We first consider a few conditions and concepts that will be instrumental for establishing our results.

2.1. The extrinsic balls and the curvature bounds. We consider a properly immersed $m$-dimensional submanifold $P^{m}$ in a complete Riemannian manifold $N^{n}$. Let $p$ denote a point in $P$ and assume that $p$ is a pole of the ambient manifold $N$. We denote the distance function from $p$ in $N^{n}$ by $r(x)=\operatorname{dist}_{N}(p, x)$ for all $x \in N$. Since $p$ is a pole there is - by definition - a unique geodesic from $x$ to $p$ which realizes the distance $r(x)$. We also denote by $r$ the restriction $\left.r\right|_{P}: P \longrightarrow \mathbb{R}_{+} \cup\{0\}$. This restriction is then called the extrinsic distance function from $p$ in $P^{m}$. The corresponding extrinsic metric balls of (sufficiently large) radius $R$ and center $p$ are denoted by $D_{R}(p) \subseteq P$ and defined as any connected component which contains $p$ of the set:

$$
D_{R}(p)=B_{R}(p) \cap P=\{x \in P \mid r(x)<R\},
$$

where $B_{R}(p)$ denotes the geodesic $R$-ball around the pole $p$ in $N^{n}$. The extrinsic ball $D_{R}(p)$ is a connected domain in $P^{m}$, with boundary $\partial D_{R}(p)$. Since $P^{m}$ is assumed to be unbounded in $N$ we have for every sufficiently large $R$ that $B_{R}(p) \cap P \neq P$.

We now present the curvature restrictions which constitute the geometric framework of our investigations.

Definition 2.1. Let $p$ be a point in a Riemannian manifold $M$ and let $x \in M-\{p\}$. The sectional curvature $K_{M}\left(\sigma_{x}\right)$ of the two-plane $\sigma_{x} \in T_{x} M$ is then called a $p$-radial sectional curvature of $M$ at $x$ if $\sigma_{x}$ contains the tangent vector to a minimal geodesic from $p$ to $x$. We denote these curvatures by $K_{p, M}\left(\sigma_{x}\right)$.

In order to control the mean curvatures $H_{P}(x)$ of $P^{m}$ at distance $r$ from $p$ in $N^{n}$ we introduce the following definition: 
Definition 2.2. The $p$-radial mean curvature function for $P$ in $N$ is defined in terms of the inner product of $H_{P}$ with the $N$-gradient of the distance function $r(x)$ as follows:

$$
\mathcal{C}(x)=-\left\langle\nabla^{N} r(x), H_{P}(x)\right\rangle \text { for all } x \in P .
$$

In the following definition, we are going to generalize the notion of radial mean convexity condition introduced in [MP5].

Definition 2.3. (see [MP5]) We say that the submanifold $P$ satisfies a radial mean convexity condition from below (respectively, from above) from the point $p \in P$ when there exists a radial smooth function $h(r)$, (that we call a bounding function), which satisfies one of the following inequalities

$$
\begin{aligned}
& \mathcal{C}(x) \geq h(r(x)) \text { for all } x \in P \quad \text { ( } h \text { bounds from below) } \\
& \mathcal{C}(x) \leq h(r(x)) \text { for all } x \in P \quad \text { ( } h \text { bounds from above) }
\end{aligned}
$$

The radial bounding function $h(r)$ is related to the global extrinsic geometry of the submanifold. For example, it is obvious that minimal submanifolds satisfy a radial mean convexity condition from above and from below, with bounding function $h=0$. On the other hand, it can be proved, see the works [Sp], [DCW], [Pa1] and [MP5], that when the submanifold is a convex hypersurface, then the constant function $h(r)=0$ is a radial bounding function from below.

The final notion needed to describe our comparison setting is the idea of radial tangency. If we denote by $\nabla^{N} r$ and $\nabla^{P} r$ the gradients of $r$ in $N$ and $P$ respectively, then we have the following basic relation:

$$
\nabla^{N} r=\nabla^{P} r+\left(\nabla^{N} r\right)^{\perp},
$$

where $\left(\nabla^{N} r\right)^{\perp}(q)$ is perpendicular to $T_{q} P$ for all $q \in P$.

When the submanifold $P$ is totally geodesic, then $\nabla^{N} r=\nabla^{P} r$ in all points, and, hence, $\left\|\nabla^{P} r\right\|=1$. On the other hand, and given the starting point $p \in P$, from which we are measuring the distance $r$, we know that $\nabla^{N} r(p)=\nabla^{P} r(p)$, so $\left\|\nabla^{P} r(p)\right\|=1$. Therefore, the difference $1-\left\|\nabla^{P} r\right\|$ quantifies the radial detour of the submanifold with respect the ambient manifold as seen from the pole $p$. To control this detour locally, we apply the following

Definition 2.4. We say that the submanifold $P$ satisfies a radial tangency condition at $p \in P$ when we have a smooth positive function

$$
g: P \mapsto \mathbb{R}_{+},
$$

so that

$$
\mathcal{T}(x)=\left\|\nabla^{P} r(x)\right\| \geq g(r(x))>0 \text { for all } \quad x \in P .
$$


Remark a. Of course, we always have

$$
\mathcal{T}(x)=\left\|\nabla^{P} r(x)\right\| \leq 1 \text { for all } x \in P .
$$

2.2. Model Spaces. As mentioned previously, the model spaces $M_{w}^{m}$ serve foremost as comparison controllers for the radial sectional curvatures of $N^{n}$.

Definition 2.5 (See [Gri], [GreW]). A $w$-model $M_{w}^{m}$ is a smooth warped product with base $B^{1}=\left[0, R[\subset \mathbb{R}\right.$ (where $0<R \leq \infty)$, fiber $F^{m-1}=$ $S_{1}^{m-1}$ (i.e. the unit $(m-1)$-sphere with standard metric), and warping function $w:\left[0, R\left[\rightarrow \mathbb{R}_{+} \cup\{0\}\right.\right.$ with $w(0)=0, w^{\prime}(0)=1$, and $w(r)>0$ for all $r>0$. The point $p_{w}=\pi^{-1}(0)$, where $\pi$ denotes the projection onto $B^{1}$, is called the center point of the model space. If $R=\infty$, then $p_{w}$ is a pole of $M_{w}^{m}$.

Remark b. The simply connected space forms $\mathbb{K}^{m}(b)$ of constant curvature $b$ can be constructed as $w$-models with any given point as center point using the warping functions

$$
w(r)=Q_{b}(r)=\left\{\begin{array}{ll}
\frac{1}{\sqrt{b}} \sin (\sqrt{b} r) & \text { if } b>0 \\
r & \text { if } b=0 \\
\frac{1}{\sqrt{-b}} \sinh (\sqrt{-b} r) & \text { if } b<0
\end{array} .\right.
$$

Note that for $b>0$ the function $Q_{b}(r)$ admits a smooth extension to $r=$ $\pi / \sqrt{b}$. For $b \leq 0$ any center point is a pole.

In the papers [O'N], [GreW], [Gri], [MP3] and [MP4], we have a complete description of these model spaces, including the computation of their sectional curvatures $K_{p_{w}, M_{w}}$ in the radial directions from the center point. They are determined by the radial function $K_{p_{w}, M_{w}}\left(\sigma_{x}\right)=K_{w}(r)=-\frac{w^{\prime \prime}(r)}{w(r)}$. Moreover, the mean curvature of the distance sphere of radius $r$ from the center point is

$$
\eta_{w}(r)=\frac{w^{\prime}(r)}{w(r)}=\frac{d}{d r} \ln (w(r))
$$

In particular, in [MP4] we introduced, for any given warping function $w(r)$, the isoperimetric quotient function $q_{w}(r)$ for the corresponding $w$-model space $M_{w}^{m}$ as follows:

$$
q_{w}(r)=\frac{\operatorname{Vol}\left(B_{r}^{w}\right)}{\operatorname{Vol}\left(S_{r}^{w}\right)}=\frac{\int_{0}^{r} w^{m-1}(t) d t}{w^{m-1}(r)} .
$$

Then, we have the following result concerning the mean exit time function and the torsional rigidity of a geodesic $R$-ball $B_{R}^{w} \subseteq M_{w}^{m}$ in terms of $q_{w}$, see [MP4]: 
Proposition 2.6. Let $E_{R}^{w}$ be the solution of the Poisson Problem (1.2), defined on the geodesic $R$-ball $B_{R}^{w}$ in the model space $M_{w}^{m}$.

Then

$$
E_{R}^{w}(r)=\int_{r}^{R} q_{w}(t) d t
$$

and

$$
\mathcal{A}_{1}\left(B_{R}^{w}\right)=\int_{B_{R}^{w}} E_{R}^{w} d \tilde{\sigma}=V_{0} \int_{0}^{R} w^{m-1}(r)\left(\int_{r}^{R} q_{w}(t) d t\right) d r,
$$

where $V_{0}$ is the volume of the unit sphere $S_{1}^{m-1}$. Differentiating with respect to $R$ gives

$$
\frac{d}{d R} \mathcal{A}_{1}\left(B_{R}^{w}\right)=q_{w}^{2}(R) \operatorname{Vol}\left(S_{R}^{w}\right)
$$

and an integration of the latter equality, gives us the following alternative expression for the torsional rigidity:

$$
\mathcal{A}_{1}\left(B_{R}^{w}\right)=\int_{B_{R}^{w}} q_{w}^{2} d \tilde{\sigma}
$$

Remark c. Since $q_{w}(r)>0$, it follows from (2.8) that for fixed $r$, the mean exit time function $E_{R}^{w}(r)$ is an increasing function of $R$. Furthermore, if $q_{w}^{\prime}(r) \geq 0$, then the average mean exit time $\mathcal{A}_{1}\left(B_{r}^{w}\right) / \operatorname{Vol}\left(B_{r}^{w}\right)$ is also a non-decreasing function of $r$.

2.3. The Isoperimetric Comparison space. Given the bounding functions $g(r), h(r)$ and the ambient curvature controller function $w(r)$ described is Subsections 2.1 and 2.2, we construct a new model space $C_{w, g, h}^{m}$, which eventually will serve as the precise comparison space for the isoperimetric quotients of extrinsic balls in $P$.

Definition 2.7. Given a smooth positive function

$$
g: P \mapsto \mathbb{R}_{+},
$$

satisfying $g(0)=1$ and $g(r(x)) \leq 1$ for all $x \in P$, a 'stretching' function $s$ is defined as follows

$$
s(r)=\int_{0}^{r} \frac{1}{g(t)} d t
$$

It has a well-defined inverse $r(s)$ for $s \in[0, s(R)]$ with derivative $r^{\prime}(s)=$ $g(r(s))$. In particular $r^{\prime}(0)=g(0)=1$.

Definition 2.8 ([MP5]). The isoperimetric comparison space $C_{w, g, h}^{m}$ is the $W$-model space with base interval $B=[0, s(R)]$ and warping function $W(s)$ defined by

$$
W(s)=\Lambda^{\frac{1}{m-1}}(r(s)),
$$


where the auxiliary function $\Lambda(r)$ satisfies the following differential equation:

$$
\begin{aligned}
\frac{d}{d r}\{\Lambda(r) w(r) g(r)\} & =\Lambda(r) w(r) g(r)\left(\frac{m}{g^{2}(r)}\left(\eta_{w}(r)-h(r)\right)\right) \\
& =m \frac{\Lambda(r)}{g(r)}\left(w^{\prime}(r)-h(r) w(r)\right)
\end{aligned}
$$

and the following boundary condition:

$$
\left.\frac{d}{d r}\right|_{r=0}\left(\Lambda^{\frac{1}{m-1}}(r)\right)=1
$$

We observe, that in spite of its relatively complicated construction, $C_{w, g, h}^{m}$ is indeed a model space $M_{W}^{m}$ with a well defined pole $p_{W}$ at $s=0: W(s) \geq 0$ for all $s$ and $W(s)$ is only 0 at $s=0$, where also, because of the explicit construction in definition 2.8 and because of equation $(2.15)$ : $W^{\prime}(0)=1$.

Note that, when $g(r)=1$ for all $r$ and $h(r)=0$ for all $r$, then the stretching function $s(r)=r$ and $W(s(r))=w(r)$ for all $r$, so $C_{w, g, h}^{m}$ becomes a model space with warping function $w, M_{w}^{m}$.

Concerning the associated volume growth properties we note the following expressions for the isoperimetric quotient function:

Proposition 2.9. Let $B_{s}^{W}\left(p_{W}\right)$ denote the metric ball of radius s centered at $p_{W}$ in $C_{w, g, h}^{m}$. Then the corresponding isoperimetric quotient function is

$$
\begin{aligned}
q_{W}(s) & =\frac{\operatorname{Vol}\left(B_{s}^{W}\left(p_{W}\right)\right)}{\operatorname{Vol}\left(\partial B_{s}^{W}\left(p_{W}\right)\right)} \\
& =\frac{\int_{0}^{s} W^{m-1}(t) d t}{W^{m-1}(s)} \\
& =\frac{\int_{0}^{r(s)} \frac{\Lambda(u)}{g(u)} d u}{\Lambda(r(s))} .
\end{aligned}
$$

Remark d. When $g(r)=1$ for all $r$, the stretching function is $s(r)=$ $r$ for all $r$, and hence

$$
\begin{aligned}
q_{W}(s) & =q_{W}(r) \\
& =\frac{\operatorname{Vol}\left(B_{r}^{W}\left(p_{W}\right)\right)}{\operatorname{Vol}\left(\partial B_{r}^{W}\left(p_{W}\right)\right)}=\frac{\int_{0}^{r} \Lambda(u) d u}{\Lambda(r)} .
\end{aligned}
$$

These are the spaces where the isoperimetric bounds and the bounds on the torsional rigidity are attained. We shall refer to the $W$-model spaces $M_{W}^{m}=C_{w, g, h}^{m}$ as the isoperimetric comparison spaces. 
2.4. Balance conditions. In the paper [MP4] we imposed two further purely intrinsic conditions on the general model spaces $M_{w}^{m}$ :

Definition 2.10. A given $w$-model space $M_{w}^{m}$ is balanced from below if the following weighted isoperimetric condition is satisfied:

$$
q_{w}(r) \eta_{w}(r) \geq 1 / m \text { for all } r \geq 0,
$$

and is balanced from above if we have the inequality

$$
q_{w}(r) \eta_{w}(r) \leq 1 /(m-1) \text { for all } r \geq 0 .
$$

A model space is called totally balanced if it is balanced both from below and from above.

The model space $M_{w}^{m}$ is easily seen to be balanced from below iff

$$
\frac{d}{d r}\left(\frac{q_{w}(r)}{w(r)}\right) \leq 0 \quad \text { for all } \quad r \geq 0,
$$

and balanced from above iff

$$
\frac{d}{d r}\left(q_{w}(r)\right) \geq 0 \quad \text { for all } \quad r \geq 0 .
$$

Observation 2.11. We note that every model space of constant non-positive sectional curvature is totally balanced. In fact, for $r>0$ we have strict inequalities in both of the two balance conditions for every model space of constant negative sectional curvature. This implies in particular, that every model space which is sufficiently close to a model space of constant negative sectional curvature is itself totally balanced.

To play the comparison setting rôle in our present setting, the isoperimetric comparison spaces must satisfy similar types of balancing conditions:

Definition 2.12. The model space $M_{W}^{m}=C_{w, g, h}^{m}$ is $w$-balanced from below (with respect to the intermediary model space $M_{w}^{m}$ ) if the following holds for all $r \in[0, R]$, resp. all $s \in[0, s(R)]$ :

$$
q_{W}(s)\left(\eta_{w}(r(s))-h(r(s))\right) \geq g(r(s)) / m .
$$

Lemma 2.13. The model space $M_{W}^{m}=C_{w, g, h}^{m}$ is $w$-balanced from below iff

$$
\frac{d}{d r}\left(\frac{q_{W}(s(r))}{g(r) w(r)}\right) \leq 0
$$

Proof. A direct differentiation using (2.16) but with respect to $r$ amounts to:

$$
\begin{aligned}
& \frac{d}{d r}\left(\frac{q_{W}(s(r))}{g(r) w(r)}\right) \\
& =\frac{1}{\Lambda(r) g^{3}(r) w^{2}(r)}\left(\Lambda(r) w(r) g(r)-m\left(\int_{0}^{r} \frac{\Lambda(t)}{g(t)} d t\right)\left(w^{\prime}(r)-h(r) w(r)\right)\right)
\end{aligned}
$$


which shows that inequality (2.23) is equivalent to inequality

$$
\Lambda(r) w(r) g(r)-m\left(\int_{0}^{r} \frac{\Lambda(t)}{g(t)} d t\right)\left(w^{\prime}(r)-h(r) w(r)\right) \leq 0,
$$

which is, in turn, using (2.16), equivalent to inequality (2.22).

Remark e. In particular the $w$-balance condition from below for $M_{W}^{m}=$ $C_{w, g, h}^{m}$ implies that

$$
\eta_{w}(r)-h(r)>0
$$

Remark f. The above definition of $w$-balance condition from below for $M_{W}^{m}$ is clearly an extension of the balance condition from below as defined in [MP4, Definition 2.12]. The condition in that paper is obtained precisely when $g(r)=1$ and $h(r)=0$ for all $r \in[0, R]$ so that $r(s)=s, W(s)=$ $w(r)$, and

$$
q_{w}(r) \eta_{w}(r) \geq 1 / m
$$

We observe that the differential inequality (2.23) becomes $(2.20)$ when $g(r)=$ 1 and $h(r)=0$.

As defined previously a general $w$-model space is totally balanced if it balanced from below and from above in the sense of equations (2.20) and (2.21). In the same way, for our present purpose, an isoperimetric comparison space $M_{W}^{m}$ can be $w$-balanced from below in the sense of Definition 2.12 and, moreover, considered itself as a model space, it can be $W$-balanced from above. In fact, these two conditions are the balancing conditions which must be satisfied by the isoperimetric comparison spaces in Theorems 5.1 and 5.3. If we differentiate equation (2.16) and infer the balance conditions (2.22) and $q_{W}^{\prime}(s) \geq 0$ we get:

Lemma 2.14. Suppose that

$$
m\left(\eta_{w}(r(s))-h(r(s))\right)-g^{2}(r(s)) \eta_{w}(r(s))-g(r(s)) g^{\prime}(r(s))>0 .
$$

Then the isoperimetric comparison space $M_{W}^{m}=C_{w, g, h}^{m}$ is w-balanced from below and $W$-balanced from above if and only if

$$
\begin{aligned}
& \frac{g(r(s))}{m\left(\eta_{w}(r(s))-h(r(s))\right)} \leq q_{W}(s) \leq \\
& \frac{g(r(s))}{m\left(\eta_{w}(r(s))-h(r(s))\right)-g^{2}(r(s)) \eta_{w}(r(s))-g(r(s)) g^{\prime}(r(s))}
\end{aligned}
$$

The set of comparison spaces $M_{W}^{m}=C_{w, g, h}^{m}$ which satisfy both balance conditions in (2.28) is clearly not empty. Indeed, as was pointed out in Observation 2.11, the conditions for balance from below and balance from above (for standard $w$-model spaces $M_{w}^{m}$ ) are both open conditions on those 
warping functions which are sufficiently close to have constant negative curvature. This means that for the special cases where $h(r)=0$ and $g(r)=1$ there are warping functions $w(r)=W(r)$, which satisfy strict inequalities in (2.28). The continuity of $q_{W}(s)$ in terms of $h(r), g(r)$ and $w(r)$ then guarantees that the space of functions satisfying these inequalities (2.28) is also non-empty.

2.5. Comparison Constellations. We now present the precise settings where our main results take place, introducing the notion of comparison constellations. For that purpose we shall bound the previously introduced notions of radial curvature and tangency by the corresponding quantities attained in some special model spaces, called isoperimetric comparison spaces to be defined in the next subsection.

Definition 2.15. Let $N^{n}$ denote a complete Riemannian manifold with a pole $p$ and distance function $r=r(x)=\operatorname{dist}_{N}(p, x)$. Let $P^{m}$ denote an unbounded complete and closed submanifold in $N^{n}$. Suppose $p \in P^{m}$ and suppose that the following conditions are satisfied for all $x \in P^{m}$ with $r(x) \in[0, R]:$

(a) The $p$-radial sectional curvatures of $N$ are bounded from below by the $p_{w}$-radial sectional curvatures of of the $w$-model space $M_{w}^{m}$ :

$$
\mathcal{K}\left(\sigma_{x}\right) \geq-\frac{w^{\prime \prime}(r(x))}{w(r(x))} .
$$

(b) The $p$-radial mean curvature of $P$ is bounded from below by a smooth radial function $h(r)$, ( $h$ is a radial convexity function):

$$
\mathcal{C}(x) \geq h(r(x)) \text {. }
$$

(c) The submanifold $P$ satisfies a radial tangency condition at $p \in P$, with smooth positive function $g$ i.e. we have a smooth positive function

$$
g: P \mapsto \mathbb{R}_{+},
$$

such that

$$
\mathcal{T}(x)=\left\|\nabla^{P} r(x)\right\| \geq g(r(x))>0 \quad \text { for all } \quad x \in P .
$$

Let $C_{w, g, h}^{m}$ denote the $W$-model with the specific warping function $W$ : $\pi\left(C_{w, g, h}^{m}\right) \rightarrow \mathbb{R}_{+}$constructed in Definition 2.8, (Subsection 2.3), via $w, g$, and $h$. Then the triple $\left\{N^{n}, P^{m}, C_{w, g, h}^{m}\right\}$ is called an isoperimetric comparison constellation bounded from below on the interval $[0, R]$.

Remark g. This definiton of isoperimetric comparison constellation bounded from below was introduced in [MP5].

A "constellation bounded from above" is given by the following dual setting, (with respect to the definition above), considering the special $W$-model spaces $C_{w, g, h}^{m}$ with $g=1$ : 
Definition 2.16. Let $N^{n}$ denote a Riemannian manifold with a pole $p$ and distance function $r=r(x)=\operatorname{dist}_{N}(p, x)$. Let $P^{m}$ denote an unbounded complete and closed submanifold in $N^{n}$. Suppose the following conditions are satisfied for all $x \in P^{m}$ with $r(x) \in[0, R]$ :

(a) The $p$-radial sectional curvatures of $N$ are bounded from above by the $p_{w}$-radial sectional curvatures of the $w$-model space $M_{w}^{m}$ :

$$
\mathcal{K}\left(\sigma_{x}\right) \leq-\frac{w^{\prime \prime}(r(x))}{w(r(x))}
$$

(b) The $p$-radial mean curvature of $P$ is bounded from above by a smooth radial function $h(r)$ :

$$
\mathcal{C}(x) \leq h(r(x)) \quad \text {. }
$$

Let $C_{w, 1, h}^{m}$ denote the $W$-model with the specific warping function $W$ : $\pi\left(C_{w, 1, h}^{m}\right) \rightarrow \mathbb{R}_{+}$constructed, (in the same way as in Definition 2.15 above), in Definition 2.8 via $w, g=1$, and $h$. Then the triple $\left\{N^{n}, P^{m}, C_{w, 1, h}^{m}\right\}$ is called an isoperimetric comparison constellation bounded from above on the interval $[0, R]$.

Remark h. The isoperimetric comparison constellations bounded from above constitutes a generalization of the triples $\left\{N^{n}, P^{m}, M_{w}^{m}\right\}$ considered in the main theorem of [MP4]. This generalization is given by the fact that we construct the isoperimetric comparison space $C_{w, g, h}^{m}$ with $g=1$, (by definition), and, when $P$ is minimal, then we consider as the bounding funtion $h=0$. It is straigthforward to see that, under these restrictions, $W=w$ and hence, $C_{w, 1,0}^{m}=M_{w}^{m}$.

\section{ISOPERIMETRIC RESULTS}

We find upper bounds for the isoperimetric quotient defined as the volume of the extrinsic sphere divided by the volume of the extrinsic ball, in the setting given by the comparison constellations. In order to do that, we need the following Laplacian comparison Theorem for manifolds with a pole (see [GreW], [JK], [MP3], [MP4], [MP5] and [MM] for more details). Moreover, we shall assume along this Section that all extrinsic balls are precompact.

Theorem 3.1. Let $N^{n}$ be a manifold with a pole $p$, let $M_{w}^{m}$ denote a $w$-model with center $p_{w}$. Then we have the following dual Laplacian inequalities for modified distance functions:

(i) Suppose that every p-radial sectional curvature at $x \in N-\{p\}$ is bounded by the $p_{w}$-radial sectional curvatures in $M_{w}^{m}$ as follows:

$$
\mathcal{K}(\sigma(x))=K_{p, N}\left(\sigma_{x}\right) \geq-\frac{w^{\prime \prime}(r)}{w(r)} .
$$


Then we have for every smooth function $f(r)$ with $f^{\prime}(r) \leq 0$ for all $r$, (respectively $f^{\prime}(r) \geq 0$ for all $\left.r\right)$ :

$$
\begin{aligned}
\Delta^{P}(f \circ r) \geq(\leq) & \left(f^{\prime \prime}(r)-f^{\prime}(r) \eta_{w}(r)\right)\left\|\nabla^{P} r\right\|^{2} \\
& +m f^{\prime}(r)\left(\eta_{w}(r)+\left\langle\nabla^{N} r, H_{P}\right\rangle\right),
\end{aligned}
$$

where $H_{P}$ denotes the mean curvature vector of $P$ in $N$.

(ii) Suppose that every p-radial sectional curvature at $x \in N-\{p\}$ is bounded by the $p_{w}$-radial sectional curvatures in $M_{w}^{m}$ as follows:

$$
\mathcal{K}(\sigma(x))=K_{p, N}\left(\sigma_{x}\right) \leq-\frac{w^{\prime \prime}(r)}{w(r)} .
$$

Then we have for every smooth function $f(r)$ with $f^{\prime}(r) \leq 0$ for all $r$, (respectively $f^{\prime}(r) \geq 0$ for all $\left.r\right)$ :

$$
\begin{aligned}
\Delta^{P}(f \circ r) \leq(\geq) & \left(f^{\prime \prime}(r)-f^{\prime}(r) \eta_{w}(r)\right)\left\|\nabla^{P} r\right\|^{2} \\
& +m f^{\prime}(r)\left(\eta_{w}(r)+\left\langle\nabla^{N} r, H_{P}\right\rangle\right),
\end{aligned}
$$

where $H_{P}$ denotes the mean curvature vector of $P$ in $N$.

The isoperimetric inequality (3.5) below has been stated and proved previously in [MP5, Theorem 7.1]. On the other hand, the isoperimetric inequality (3.6) has been stated and proved in [MP4], but only under the assumption that $P$ is minimal and that the model space satisfies a more restrictive balance condition, see Remark f. For completeness we therefore give a sketch of the proof of inequality (3.6) below.

Theorem 3.2. There are two dual settings to be considered:

(i) Consider an isoperimetric comparison constellation bounded from below $\left\{N^{n}, P^{m}, C_{w, g, h}^{m}\right\}$. Assume that the isoperimetric comparison space $C_{w, g, h}^{m}$ is $w$-balanced from below. Then

$$
\frac{\operatorname{Vol}\left(\partial D_{R}\right)}{\operatorname{Vol}\left(D_{R}\right)} \leq \frac{\operatorname{Vol}\left(\partial B_{s(R)}^{W}\right)}{\operatorname{Vol}\left(B_{s(R)}^{W}\right)} \leq \frac{m}{g(R)}\left(\eta_{w}(R)-h(R)\right) .
$$

where $s(R)$ is the stretched radius given by Definition 2.7.

(ii) Consider an isoperimetric comparison constellation bounded from above $\left\{N^{n}, P^{m}, C_{w, 1, h}^{m}\right\}$. Assume that the isoperimetric comparison space $C_{w, 1, h}^{m}$ is w-balanced from below. Then

$$
\frac{\operatorname{Vol}\left(\partial D_{R}\right)}{\operatorname{Vol}\left(D_{R}\right)} \geq \frac{\operatorname{Vol}\left(\partial B_{R}^{W}\right)}{\operatorname{Vol}\left(B_{R}^{W}\right)} .
$$

If equality holds in (3.6) for some fixed radius $R>0$, then $D_{R}$ is a cone in the ambient space $N^{n}$. 
Proof. The proof starts from the same point for both inequalities. As in [MP5], we define a second order differential operator L on functions $f$ of one real variable as follows:

$$
\mathrm{L} f(r)=f^{\prime \prime}(r) g^{2}(r)+f^{\prime}(r)\left(\left(m-g^{2}(r)\right) \eta_{w}(r)-m h(r)\right),
$$

and consider the smooth solution $\psi(r)$ to the following Dirichlet-Poisson problem:

$$
\begin{aligned}
\mathrm{L} \psi(r) & =-1 \quad \text { on }[0, R], \\
\psi(R) & =0 .
\end{aligned}
$$

The ODE is equivalent to the following:

$$
\psi^{\prime \prime}(r)+\psi^{\prime}(r)\left(-\eta_{w}(r)+\frac{m}{g^{2}(r)}\left(\eta_{w}(r)-h(r)\right)\right)=-\frac{1}{g^{2}(r)} .
$$

The solution is constructed via the auxiliary function $\Lambda(r)$ from equation (2.14) and it is given, as it can be seen in [MP5], by:

$$
\begin{aligned}
\psi^{\prime}(r) & =\Gamma(r)=\frac{-1}{g(r) \Lambda(r)} \int_{0}^{r} \frac{\Lambda(t)}{g(t)} d t \\
& =-\frac{\operatorname{Vol}\left(B_{s(r)}^{W}\right)}{g(r) \operatorname{Vol}\left(\partial B_{s(r)}^{W}\right)}=-\frac{q_{W}(s(r))}{g(r)}
\end{aligned}
$$

and then

$$
\begin{aligned}
\psi(r) & =\int_{r}^{R} \frac{1}{g(u) \Lambda(u)}\left(\int_{0}^{u} \frac{\Lambda(t)}{g(t)} d t\right) d u \\
& =\int_{r}^{R} \frac{q_{W}(s(u))}{g(u)} d u=\int_{s(r)}^{s(R)} q_{W}(t) d t .
\end{aligned}
$$

We must recall, as it was pointed out in Remark d, that, when we consider a comparison constellation bounded from above, as in the statement (ii) of the Theorem, then $g(r)=1$ in (3.7) and (3.9), so $s(r)=r$, and

$$
\psi^{\prime}(r)=-q_{W}(r)=-\frac{\operatorname{Vol}\left(B_{r}^{W}\right)}{\operatorname{Vol}\left(S_{r}^{W}\right)} .
$$

Then - because of the balance condition (2.22) and equation (3.9) - the function $\psi(r)$ enjoys the following inequality:

$$
\psi^{\prime \prime}(r)-\psi^{\prime}(r) \eta_{w}(r) \geq 0
$$

The second common step to prove isoperimetric inequalities (3.5) and (3.6), is to transplant $\psi(r)$ to $D_{R}$ defining

$$
\psi: D_{R} \longrightarrow \mathbb{R} ; \quad \psi(x):=\psi(r(x)) .
$$

Now, we are going to focus attention on the isoperimetric inequality (3.6). In this case, we have that the sectional curvatures of the ambient manifold 
are bounded from above, inequality (3.12), that the p-radial mean curvature of $P$ is bounded from above by $h(r)$, and that $\eta_{w}(r)-h(r)>0$ for all $r>0$. Then, applying now the Laplace inequality (3.4) in Theorem 3.1 for the transplanted function $\psi(r)$ we have the following comparison,

$$
\begin{aligned}
\Delta^{P} \psi(r(x)) \leq & \left(\psi^{\prime \prime}(r(x))-\psi^{\prime}(r(x)) \eta_{w}(r(x))\right)\left\|\nabla^{P} r\right\|^{2} \\
& +m \psi^{\prime}(r(x))\left(\eta_{w}(r(x))-h(r(x))\right) \\
\leq & \operatorname{L} \psi(r(x))=-1=\Delta^{P} E(x) .
\end{aligned}
$$

Applying the divergence theorem, using the unit normal $\nabla^{P} r /\left\|\nabla^{P} r\right\|$ to $\partial D_{r}$, we get, as in [Pa1], but now for submanifolds with $p$-radial mean curvature bounded from above by $h(r)$ :

$$
\begin{aligned}
\operatorname{Vol}\left(D_{R}\right) & \leq \int_{D_{R}}-\Delta^{P} \psi(r(x)) d \sigma \\
& =-\Gamma(R) \int_{\partial D_{R}}\left\|\nabla^{P} r\right\| d \sigma \\
& \leq-\Gamma(R) \operatorname{Vol}\left(\partial D_{R}\right) .
\end{aligned}
$$

which shows the isoperimetric inequality (3.6), because in this case, and in view of remark d, we have that

$$
\Gamma(r)=\psi^{\prime}(r)=-q_{W}(r)=-\frac{\operatorname{Vol}\left(B_{r}^{W}\right)}{\operatorname{Vol}\left(S_{r}^{W}\right)} .
$$

To prove the equality assertion, we note that equality in (3.6) for some fixed $R>0$ implies that the inequalities in (3.13) and (3.14) become equalities. Hence, $\left\|\nabla^{P} r\right\|=1=\left\|\nabla^{N} r\right\|$ in $D_{R}$, so $\nabla^{P} r=\nabla^{N} r$ in $D_{R}$. Then, all the geodesics in $N$ starting at $p$ thus lie in $P$, so $D_{R}=\exp _{p}\left(\widetilde{D}_{R}\right)$, with $\widetilde{D}_{R}$ being the 0 -centered $R$-ball in $T_{p} P$. Therefore, $D_{R}$ is a cone in $N$.

Inequality (3.5) is proved in the same way, see [MP5], but using the Laplace inequality (3.2) to the transplanted function $\psi(r)$. In this case, we are assuming that the sectional curvatures of the ambient manifold are bounded from below and the $p$-radial mean curvature of the submanifold is bounded from below by the function $h(r)$. Under these conditions, we have

$$
\Delta^{P} \psi(r(x)) \geq \mathrm{L} \psi(r(x))=-1=\Delta^{P} E(x) .
$$

Then, we obtain the result applying the divergence theorem as before and taking into account that in this case the derivative of $\psi(r)$ is

$$
\Gamma(R)=\psi^{\prime}(R)=-\frac{\operatorname{Vol}\left(B_{s(R)}^{W}\right)}{g(R) \operatorname{Vol}\left(\partial B_{s(R)}^{W}\right)} .
$$


A corollary of the proof of Theorem 3.2 is the following

Proposition 3.3. Let us consider the isoperimetric model space $M_{W}^{m}=$ $C_{w, g, h}^{m}$. Then

$$
\psi(r)=E_{s(R)}^{W}(s(r)) \text { for all } r \in[0, R],
$$

where $s$ is the stretching function defined in equation (2.12) and

$$
E_{s(R)}^{W}: B_{s(R)}^{W} \longrightarrow \mathbb{R}
$$

is the solution of the Poisson problem

$$
\begin{aligned}
\Delta^{C_{w, g, h}^{m}} E(s) & =-1 \quad \text { on } \quad B_{s(R)}^{W} \quad, \\
E & =0 \quad \text { on } \partial B_{s(R)}^{W} .
\end{aligned}
$$

Proof. This follows directly from Proposition 2.6 by applying (3.11).

The proof of the next Corollary 3.4, (where we assume that the submanifold $P$ has bounded $p$-radial mean curvature from above or from below), follows the same formal steps as the corresponding results for minimal submanifolds, which can be founded in [MP5], [Pa2], and in [MP4]. As in these proofs, the co-area formula, see [Ch1], plays here a fundamental rôle.

Corollary 3.4. Again we consider the two dual settings:

(i) Let $\left\{N^{n}, P^{m}, C_{w, q, h}^{m}\right\}$ be a comparison constellation bounded from below on the interval $[0, R]$, as in statement (i) of Theorem 3.2.

Then

$$
\operatorname{Vol}\left(D_{r}\right) \leq \operatorname{Vol}\left(B_{s(r)}^{W}\right) \quad \text { for every } r \in[0, R] .
$$

(ii) Let $\left\{N^{n}, P^{m}, C_{w, 1, h}^{m}\right\}$ be a comparison constellation bounded from above on the interval $[0, R]$, as in statement (ii) of Theorem 3.2.

Then

$$
\operatorname{Vol}\left(D_{r}\right) \geq \operatorname{Vol}\left(B_{r}^{W}\right) \quad \text { for every } r \in[0, R] .
$$

Equality in (3.18), for all $r \in[0, R]$ and some fixed radius $R>0 \mathrm{im}$ plies that $D_{R}$ is a cone in $N^{n}$, using the same arguments as in the proof of Theorem 3.2.

\section{Symmetrization into model spaces}

As in [MP4] we use the concept of Schwarz-symmetrization as considered in e.g. [Ba], [Po], or, more recently, in [Mc] and [Ch2]. We review some facts about this instrumental tool. 
Definition 4.1. Suppose $D$ is a precompact open connected domain in $P^{m}$. Then the $w$-model space symmetrization of $D$ is denoted by $D^{*}$ and is defined to be the unique $p_{w}$-centered ball $D^{*}=B^{w}(D)$ in $M_{w}^{m}$ satisfying $\operatorname{Vol}(D)=\operatorname{Vol}\left(B^{w}(D)\right)$. In the particular case where $D$ is actually an extrinsic metric ball $D_{R}$ in $P$ of radius $R$ we may write

$$
D_{R}^{*}=B^{w}(D)=B_{T(R)}^{w},
$$

where $T(R)$ is some increasing function of $R$ which depends on the geometry of $P$, according to the defining property:

$$
\operatorname{Vol}\left(D_{R}\right)=\operatorname{Vol}\left(B_{T(R)}^{w}\right)
$$

We also introduce the notion of a symmetrized function on the symmetrization $D^{*}$ of $D$ as follows.

Definition 4.2. Let $f$ denote a nonnegative function on $D$

$$
f: D \subseteq P \rightarrow \mathbb{R}^{+} \cup\{0\} .
$$

For $t>0$ we let

$$
D(t)=\{x \in D \mid f(x) \geq t\} .
$$

Then the symmetrization of $f$ is the function $f^{*}: D^{*} \rightarrow \mathbb{R} \cup\{0\}$ defined by

$$
f^{*}\left(x^{*}\right)=\sup \left\{t \mid x^{*} \in D(t)^{*}\right\} .
$$

Proposition 4.3. The symmetrized objects $f^{*}$ and $D^{*}$ satisfy the following properties:

(1) The function $f^{*}$ depends only on the geodesic distance to the center $p_{w}$ of the ball $D^{*}$ in $M_{w}^{m}$ and is non-increasing.

(2) The functions $f$ and $f^{*}$ are equimeasurable in the sense that

$$
\operatorname{Vol}_{P}(\{x \in D \mid f(x) \geq t\})=\operatorname{Vol}_{M_{w}^{m}}\left(\left\{x^{*} \in D^{*} \mid f^{*}\left(x^{*}\right) \geq t\right\}\right)
$$

for all $t \geq 0$. In particular, for all $t>0$, we have

$$
\int_{D(t)} f d \sigma \leq \int_{D(t)^{*}} f^{*} d \tilde{\sigma}
$$

Remark i. The proof of these properties follows the proof of the classical Schwarz symmetrization using the 'slicing' technique for symmetrized volume integrations and comparison - see e.g. [Ch2].

In the proof of both Theorem 5.1 and Theorem 5.3 in Section 5, we shall consider a symmetric model space rearrangement of the extrinsic ball $D_{R}$ as it has been described in Definition 4.1 and Definition 4.2 , namely, a symmetrization of $D_{R}$ which is a geodesic $T(R)$-ball in the model space $M_{W}^{m}$ such that $\operatorname{vol}\left(D_{R}\right)=\operatorname{vol}\left(B_{T(R)}^{W}\right)$, together the symmetrization of the transplanted radial function $\psi: D_{R} \longrightarrow \mathbb{R}$ of the solution of the Poisson problem (3.8) in $[0, R]$. We know (see Proposition 3.3) that $\psi(r)=E_{s(R)}^{W}\left(s(r)\right.$ ), where $E_{s(R)}^{W}$ 
is the solution of the Poisson problem (3.16).

This symmetrization is a function $\psi^{*}: B_{T(R)}^{W} \longrightarrow \mathbb{R}$ which satisfies the property that inequality (4.2) becomes an equality. This property becomes a crucial fact in the proof of Theorems 5.1 and 5.3.

Theorem 4.4. Let $\psi^{*}: B_{T(R)}^{W} \longrightarrow \mathbb{R}$ be the symmetrization of the transplanted radial function $\psi: D_{R} \longrightarrow \mathbb{R}$ of the solution of the Poisson problem (3.8) in $[0, R]$. Then

$$
\int_{D_{R}} \psi d \sigma=\int_{B_{T(R)}^{W}} \psi^{*} d \tilde{\sigma}
$$

Proof. First of all, we are going to define $\psi^{*}$. To do that, let us consider $T=\max _{[0, R]} \psi$. On the other hand, and given $t \in[0, T]$, let us define the sets

$$
D(t)=\left\{x \in D_{R} \mid \psi(r(x)) \geq t\right\} \quad,
$$

and

$$
\Gamma(t)=\left\{x \in D_{R} \mid \psi(r(x))=t\right\} .
$$

As $\psi(r(x))=E_{s(R)}^{W}(s(r(x)))$ for all $x \in D_{R}$, then $\psi$ is radial and nonincreasing, its maximum $T$ will be attained at $r=0, D(t)$ is the extrinsic ball in $P$ with radius $a(t):=\psi^{-1}(t)$, (we denote it as $D_{a(t)}$ ), and $\Gamma(t)$ is its boundary, the extrinsic sphere with radius $a(t), \partial D_{a(t)}$. We have too that $D(0)=D_{R}$ and $D(T)=\{p\}$, the center of the extrinsic ball $D_{R}$.

We consider the symmetrizations of the sets $D(t) \subseteq P$, namely, the geodesic balls $D(t)^{*}=B_{\tilde{r}(t)}^{W}$ in $M_{W}^{m}$ such that

$$
\operatorname{Vol}(D(t))=\operatorname{Vol}\left(D_{a(t)}\right)=\operatorname{Vol}\left(B_{\tilde{r}(t)}^{W}\right) \quad .
$$

Hence, we have defined a non-increasing function

$$
\tilde{r}:[0, T] \longrightarrow[0, T(R)] ; \tilde{r}=\tilde{r}(t) \quad,
$$

defined as the radius $\tilde{r}(t)$ from the center $\tilde{p}$ of the model space $C_{w, g, h}^{m}$ such that $\operatorname{Vol}\left(B_{\tilde{r}(t)}^{W}\right)=\operatorname{Vol}(D(t))=\operatorname{Vol}\left(D_{a(t)}\right)$, (and hence, $\tilde{r}(0)=T(R)$ and $\tilde{r}(T)=0)$, with inverse

$$
\phi:[0, T(R)] \longrightarrow[0, T] ; \phi=\phi(\tilde{r}),
$$

such that $\phi^{\prime}(\tilde{r}(t))=\frac{1}{\tilde{r}^{\prime}(t)}$ for all $t \in[0, T]$.

Thus, given $\tilde{x} \in B_{T(R)}^{W}$, and taking into account that

$$
B_{T(R)}^{W}=\cup_{t \in[0, T]} \partial D(t)^{*}=\cup_{t \in[0, T]} S_{\tilde{r}(t)}^{W},
$$

there exists some biggest value $t_{0}$ such that $r_{\tilde{p}}(\tilde{x})=\tilde{r}\left(t_{0}\right)$, (and hence, $\tilde{x} \in$ $\left.D\left(t_{0}\right)^{*}\right)$. Therefore, in accordance with Definition 4.2 , the symmetrization 
of $\psi: D_{R} \longrightarrow \mathbb{R}$ is a function $\psi^{*}: B_{T(R)}^{W} \longrightarrow \mathbb{R}$ defined as

$$
\psi^{*}(\tilde{x})=E_{s(R)}^{W *}\left(s\left(r_{\tilde{p}}(\tilde{x})\right)=t_{0}=\phi\left(\tilde{r}\left(t_{0}\right)\right) .\right.
$$

Remark j. We pause to make two observations:

(i) Note that $\psi^{*}$ is a radial function, $\psi^{*}(\tilde{x})=\psi^{*}(\tilde{r}(\tilde{x}))=\psi^{*}(\tilde{r})$. Therefore, for all $\tilde{r} \in[0, T(R)]$ and $t \in[0, T]$, we have

$$
\psi^{*^{\prime}}(\tilde{r})=\phi^{\prime}(\tilde{r}(t))=\frac{1}{\tilde{r}^{\prime}(t)} .
$$

(ii) Let $T(R)$ be the radius such that $\operatorname{Vol}\left(B_{T(R)}^{W}\right)=\operatorname{Vol}\left(D_{R}\right)$, and let $s(R)$ be the "stretched" radius $s(R)=\int_{0}^{R} \frac{1}{g(t)} d t$.

As the comparison constellation is bounded from below, and by virtue of inequality (3.17) in Corollary 3.4, we have, for all $t \in[0, T], \operatorname{Vol}\left(B_{\tilde{r}(t)}^{W}\right)=$ $\operatorname{Vol}\left(D_{a(t)}\right) \leq \operatorname{Vol}\left(B_{s(a(t))}^{W}\right)$, so $\tilde{r}(t) \leq s(a(t))$ for all $t \in[0, T]$ and then

$$
T(R)=b(0) \leq s(a(0))=s(R) \quad .
$$

By definition of $\psi^{*}$, we have $\psi^{*}=\phi \circ \tilde{r}$ on $B_{T(R)}^{W}$, see (4.4). Then, using the formula for integration in a disc in a model space ([Ch1, p. 47]) we get

$$
\begin{aligned}
\int_{B_{T(R)}^{W}} \psi^{*} d \tilde{\sigma} & =\int_{B_{T(R)}^{W}} \phi \circ \tilde{r} d \tilde{\sigma} \\
& =\int_{S_{1}^{0, m-1}} d A(\xi)\left\{\int_{0}^{T(R)} \phi(\tilde{r}) W^{m-1}(\tilde{r}) d \tilde{r}\right\} \\
& =\int_{0}^{T(R)} \phi(\tilde{r}) \operatorname{Vol}\left(S_{1}^{0, m-1}\right) W^{m-1}(\tilde{r}) d \tilde{r} \\
& =\int_{0}^{T(R)} \phi(\tilde{r}) \operatorname{Vol}\left(S_{\tilde{r}}^{W}\right) d \tilde{r} .
\end{aligned}
$$

Now, we change the variable using the bijective, (monotone decreasing), function $\tilde{r}:[0, T] \longrightarrow[0, T(R)] ; \tilde{r}(0)=T(R), \tilde{r}(T)=0$, so

$$
\int_{0}^{T(R)} \phi(\tilde{r}) \operatorname{Vol}\left(S_{\tilde{r}}^{W}\right) d \tilde{r}=\int_{T}^{0} \phi(\tilde{r}(t)) \operatorname{Vol}\left(S_{\tilde{r}(t)}^{W}\right) \tilde{r}^{\prime}(t) d t
$$

But we know that $\phi(\tilde{r}(t))=t$ for all $t \in[0, T]$, and, on the other hand, denoting as $V(t)=\operatorname{Vol}\left(B_{\tilde{r}(t)}^{W}\right)=\operatorname{Vol}(D(t))$ for all $t \in[0, T]$, we have

$$
V^{\prime}(t)=\operatorname{Vol}\left(S_{\tilde{r}(t)}^{W}\right) \tilde{r}^{\prime}(t) \text { for all } t \in[0, T],
$$

and hence

$$
\int_{T}^{0} \phi(\tilde{r}(t)) \operatorname{Vol}\left(S_{\tilde{r}(t)}^{W}\right) \tilde{r}^{\prime}(t) d t=-\int_{0}^{T} t V^{\prime}(t) d t
$$


Now, we apply co-area formula to the following setting: we have the transplanted function $\psi: D_{R} \longrightarrow \mathbb{R}$, and the sets $D(t)$, with their boundaries $\Gamma(t)$. We have, by definition, that $V(t)=\operatorname{Vol}(D(t))$, so

$$
V^{\prime}(t)=-\int_{\Gamma(t)}\left\|\nabla^{P} \psi\right\|^{-1} d \sigma_{t}
$$

Hence, putting together all the equalities before, taking into account that $\left.\psi\right|_{\Gamma(t)}=t$ for all $t \in[0, T]$, and using the co-area formula again ([Ch1, equation (4) in Theorem 1, p. 86]), we conclude

$$
\int_{B_{T(R)}^{W}} \psi^{*} d \tilde{\sigma}=-\int_{0}^{T} t V^{\prime}(t) d t=\int_{D_{R}} \psi d \sigma .
$$

\section{MAin Results}

By definition, the torsional rigidity $\mathcal{A}_{1}\left(D_{R}\right)$ is the $D_{R}$-integral of the mean exit time function $E_{R}(x)$ from $x$ in $D_{R}$. We note that for most minimally immersed submanifolds $P^{m}$ in the flat Euclidean spaces $\mathbb{R}^{n}$ with the obvious choice of comparison model space, $M_{W}^{m}=\mathbb{R}^{m}, W(r)=r$, we have (see [Ma1], [Pa2]):

$$
\begin{aligned}
& E_{R}(x)=E_{R}^{W}(r(x)) \text { for all } x \in D_{R}, \\
& \text { but also } \operatorname{Vol}\left(D_{R}\right)>\operatorname{Vol}\left(B_{R}^{W}\right), \\
& \text { so that } \mathcal{A}_{1}\left(D_{R}\right)>\mathcal{A}_{1}\left(B_{R}^{W}\right) .
\end{aligned}
$$

In this sense Theorem 5.1 is a generalization of this fact, when we assume that the ambient space has sectional curvatures bounded from below, and that the mean curvature of the submanifold is controlled along the radial directions from the pole. These assumptions includes minimality and convexity of the submanifold. This result is based on previous geometrical and analytical considerations from [MP5].

Theorem 5.1. Let $\left\{N^{n}, P^{m}, C_{w, g, h}^{m}\right\}$ denote a comparison constellation bounded from below in the sense of Definition 2.15. Assume that $M_{W}^{m}=C_{w, g, h}^{m}$ is $w$-balanced from below, and $W$-balanced from above. Let $D_{R}$ be a precompact extrinsic $R$-ball in $P^{m}$, with center at a point $p \in P$ which also serves as a pole in $N$. Then

$$
\mathcal{A}_{1}\left(D_{R}\right) \geq \mathcal{A}_{1}\left(B_{T(R)}^{W}\right),
$$

where $B_{T(R)}^{W}$ is the Schwarz symmetrization of $D_{R}$ in the $W$-model space $C_{w, g, h}^{m}$, i.e., it is the geodesic ball in $C_{w, g, h}^{m}$ such that $\operatorname{Vol}\left(D_{R}\right)=\operatorname{Vol}\left(B_{T(R)}^{W}\right)$. 
Proof of Theorem 5.1. Given the solution $E_{R}$ to the Dirichlet-Poisson equation on $D_{R}$, we compare it with the transplanted function $\psi(r(x))$, defined on $D_{R}$ as the radial solution of equation (3.8) in the proof of Theorem 3.2. In fact, by inequality (3.15) we have that $\psi-E_{R}$ is a subharmonic function with $E_{R}(R)=\psi(R)=0$, so, applying Maximun Principle,

$$
E_{R} \geq \psi \text { on } D_{R} \text {. }
$$

Using this inequality and Proposition 4.4, we have

$$
\mathcal{A}_{1}\left(D_{R}\right)=\int_{D_{R}} E_{R} d \sigma \geq \int_{D_{R}} \psi d \sigma=\int_{B_{T(R)}^{W}} \psi^{*} d \tilde{\sigma} .
$$

The symmetrized function $\psi^{*}: B_{T(R)}^{W} \longrightarrow \mathbb{R}$ is a radial function, but it does not necessarily satisfy the Poisson equation on $B_{T(R)}^{W}$. Then, we are going to compare $\psi^{*}$ with the radial solution $E_{T(R)}^{W}: B_{T(R)}^{W} \longrightarrow \mathbb{R}$ of the Dirichlet-Poisson problem

$$
\begin{aligned}
\Delta^{C} C_{w, g, h}^{m} E & =-1 \quad \text { on } \quad B_{T(R)}^{W} \\
E & =0 \text { on } \partial B_{T(R)}^{W} .
\end{aligned}
$$

To do that, we shall prove the following (the proof is given below after finishing the proof of Theorem 5.1)

\section{Proposition 5.2.}

$$
\psi^{*^{\prime}}(\tilde{r}) \leq E_{T(R)}^{W^{\prime}}(\tilde{r}) \text { for all } \tilde{r} \in[0, T(R)]
$$

Assuming (5.4) for a moment, integrating from $\tilde{r}$ to $T(R)$ both sides of inequality (5.4), and taking into account that

$$
\psi^{*}(T(R))=\phi(T(R))=0=E_{T(R)}^{W}(T(R)),
$$

we obtain, for all $\tilde{r} \in[0, T(R)]$,

$$
-\psi^{*}(\tilde{r})=\int_{\tilde{r}}^{T(R)} \psi^{*^{\prime}}(l) d l \leq \int_{\tilde{r}}^{T(R)} E_{T(R)}^{W^{\prime}}(l) d l=-E_{T(R)}^{W}(\tilde{r})
$$

and hence,

Therefore,

$$
\psi^{*}(\tilde{r}) \geq E_{T(R)}^{W}(\tilde{r}) \text { for all } \tilde{r} \in[0, T(R)]
$$

$$
\begin{aligned}
\mathcal{A}_{1}\left(D_{R}\right) & =\int_{D_{R}} E_{R} d \sigma \geq \int_{D_{R}} \psi d \sigma=\int_{B_{T(R)}^{W}} \psi^{*} d \tilde{\sigma} \\
& \geq \int_{B_{T(R)}^{W}} E_{T(R)}^{W} d \tilde{\sigma}=\mathcal{A}_{1}\left(B_{T(R)}^{W}\right),
\end{aligned}
$$


and the Theorem is proved.

Proof of Proposition 5.2. Using equations (4.5), (4.9) and (4.11), we have that

$$
\psi^{*^{\prime}}(\tilde{r})=\frac{1}{\tilde{r}^{\prime}(t)}=-\frac{\operatorname{Vol}\left(S_{\tilde{r}(t)}^{W}\right)}{\int_{\Gamma(t)}\left\|\nabla^{P} \psi\right\|^{-1} d \sigma_{t}} .
$$

As $\psi(r)$ is radial, we have

$$
\left\|\nabla^{P} \psi(r)\right\|=\left|\psi^{\prime}(r)\right|\left\|\nabla^{P} r\right\| \geq\left|\psi^{\prime}(r)\right| g(r),
$$

so, as $\Gamma(t)=\partial D_{a(t)}$ for all $t \in[0, T]$, we have that

$$
\begin{aligned}
\int_{\Gamma(t)}\left\|\nabla^{P} \psi\right\|^{-1} d \sigma_{t} & =\frac{1}{\left|\psi^{\prime}(a(t))\right|} \int_{\partial D_{a(t)}}\left\|\nabla^{P} r\right\|^{-1} \\
& \leq \frac{1}{\left|\psi^{\prime}(a(t))\right| g(a(t))} \operatorname{Vol}\left(\partial D_{a(t)}\right),
\end{aligned}
$$

and hence, by equation (3.10)

$$
\begin{aligned}
\psi^{*^{\prime}}(\tilde{r}(t)) & \leq-\left|\psi^{\prime}(a(t))\right| g(a(t)) \frac{\operatorname{Vol}\left(S_{\tilde{r}(t)}^{W}\right)}{\operatorname{Vol}\left(\partial D_{a(t)}\right)} \\
& =-\frac{\operatorname{Vol}\left(B_{s(a(t))}^{W}\right)}{\operatorname{Vol}\left(S_{s(a(t))}^{W}\right)} \frac{\operatorname{Vol}\left(S_{\tilde{r}(t)}^{W}\right)}{\operatorname{Vol}\left(\partial D_{a(t)}\right)} .
\end{aligned}
$$

But we have that (see Remark $\mathrm{j}$ in the proof of Theorem 4.4 and inequality (3.17) in Corollary 3.4)

$$
\tilde{r}(t) \leq s(a(t)) \text { for all } t,
$$

so, since $q_{W}^{\prime}(r) \geq 0$, we get:

$$
\frac{\operatorname{Vol}\left(B_{\tilde{r}(t)}^{W}\right)}{\operatorname{Vol}\left(S_{\tilde{r}(t)}^{W}\right)} \leq \frac{\operatorname{Vol}\left(B_{s(a(t))}^{W}\right)}{\operatorname{Vol}\left(S_{s(a(t))}^{W}\right)} .
$$

Therefore, as $\operatorname{Vol}\left(B_{\tilde{r}(t)}^{W}\right)=\operatorname{Vol}\left(D_{a(t)}\right)$,

$$
\psi^{*^{\prime}}(\tilde{r}(t)) \leq-\frac{\operatorname{Vol}\left(D_{a(t)}\right)}{\operatorname{Vol}\left(\partial D_{a(t)}\right)} \quad .
$$

Now, we apply again the isoperimetric inequality of Theorem 3.2 (i), the fact that $\tilde{r}(t) \leq s(a(t))$ and that $q_{W}^{\prime}(r) \geq 0$ to obtain finally

$$
\begin{aligned}
\psi^{*^{\prime}}(\tilde{r}(t)) & \leq-\frac{\operatorname{Vol}\left(D_{a(t)}\right)}{\operatorname{Vol}\left(\partial D_{a(t)}\right)} \leq-\frac{\operatorname{Vol}\left(B_{s(a(t))}^{W}\right)}{\operatorname{Vol}\left(S_{s(a(t))}^{W}\right)} \\
& \leq-\frac{\operatorname{Vol}\left(B_{\tilde{r}(t)}^{W}\right)}{\operatorname{Vol}\left(S_{\tilde{r}(t)}^{W}\right)}=E_{T(R)}^{W^{\prime}}(\tilde{r}(t))
\end{aligned}
$$


Theorem 5.3 below is a generalization of the result [MP4, Theorem 2.1]. In that paper, we obtained an upper bound for the torsional rigidity of the extrinsic domains of a minimal submanifold. We assume now that the radial mean curvature of the submanifold is bounded from above, and, as in [MP4], that the ambient manifold has sectional curvatures bounded from above. Hence, we have the following generalization to submanifolds which are not necessarily minimal:

Theorem 5.3. Let $\left\{N^{n}, P^{m}, C_{w, 1, h}^{m}\right\}$ denote a comparison constellation bounded from above. Assume that $M_{W}^{m}=C_{w, 1, h}^{m}$ is w-balanced from below, $W$-balanced from above, and that it has infinite volume. Let $D_{R}$ be a precompact extrinsic $R$-ball in $P^{m}$, with center at a point $p \in P$ which also serves as a pole in $N$. Then

$$
\mathcal{A}_{1}\left(D_{R}\right) \leq \mathcal{A}_{1}\left(B_{T(R)}^{W}\right),
$$

where $B_{T(R)}^{W}$ is the Schwarz symmetrization of $D_{R}$ in the $W$-model space $M_{W}^{m}$, i.e., it is the geodesic ball in $M_{W}^{m}$ such that $\operatorname{Vol}\left(D_{R}\right)=\operatorname{Vol}\left(B_{T(R)}^{W}\right)$. Equality in (5.14) for some fixed radius $R$ implies that $D_{R}$ is a cone in $N$.

Proof of Theorem 5.3. The proof of this Theorem follows the lines of the Theorem 5.1, and the same scheme as the proof of Theorem 2.1 in [MP4]. In this proof, however, the sign of some crucial inequalities is reversed with respect the proof of Theorem 5.1. In fact, the new geometric setting given by the comparison constellation bounded from above give us inequality (3.13) so when we compare the solution of the problem (3.8) with the solution $E_{R}$ to the Dirichlet-Poisson equation on $D_{R}$, we conclude, applying too the maximum principle, that $E_{R} \leq \psi$ on $D_{R}$, and hence, using too Proposition 4.4 ,

$$
\mathcal{A}_{1}\left(D_{R}\right)=\int_{D_{R}} E_{R} d \sigma \leq \int_{D_{R}} \psi d \sigma=\int_{B_{T(R)}^{W}} \psi^{*} d \tilde{\sigma},
$$

where $B_{T(R)}^{W}$ is the symmetrization of $D_{R}$ in $C_{w, h}^{m}$.

We must remark that as the comparison constellation is bounded from above, we have, by virtue of Corollary 3.4, that

so $\tilde{r}(t) \geq a(t)$ for all $t$ and

$$
\operatorname{Vol}\left(B_{\tilde{r}(t)}^{W}\right)=\operatorname{Vol}\left(D_{a(t)}\right) \geq \operatorname{Vol}\left(B_{a(t)}^{W}\right),
$$

$$
\tilde{r}(0)=T(R) \geq a(0)=R .
$$

Now, following the lines of the proof of Theorem 5.1 we have the following, which will be proved below:

Proposition 5.4.

$$
\psi^{*^{\prime}}(\tilde{r}) \geq E_{T(R)}^{W^{\prime}}(\tilde{r}) \text { for all } \tilde{r} \in[0, T(R)] .
$$


Since

$$
\psi^{*}(T(R))=\phi(T(R))=0=E_{T(R)}^{W}(T(R)),
$$

we obtain, integrating (5.17) from $\tilde{r}$ to $T(R)$, that

$$
E_{R}^{W *}(\tilde{r}) \leq E_{T(R)}^{W}(\tilde{r}) \text { for all } \tilde{r} \in[0, T(R)] .
$$

Therefore,

$$
\begin{aligned}
\mathcal{A}_{1}\left(D_{R}\right) & =\int_{D_{R}} E_{R} d \sigma \leq \int_{D_{R}} \psi d \sigma=\int_{B_{T(R)}^{W}} \psi^{*} d \tilde{\sigma} \\
& \leq \int_{B_{T(R)}^{W}} E_{T(R)}^{W} d \tilde{\sigma}=\mathcal{A}_{1}\left(B_{T(R)}^{W}\right),
\end{aligned}
$$

and the Theorem is proved.

Proof of Proposition 5.4. This proof follows the same steps as the proof of Proposition 5.2, taking into account that in this case the comparison constellation is bounded from above, and hence, we shall use the isoperimetric inequality (3.6) in Theorem 3.2, and inequality (3.18) in Corollary 3.4, inverting all inequalities.

Remark k. The volume of the $W$-model may be finite and we need to guarantee that there is enough room for the symmetrization construction, because of inequality (5.16). For this reason, we assume that the volume of the model space is infinite. Alternatively we could assume that $W^{\prime}(r)>0$, because then the volume $\operatorname{Vol}\left(B_{r}^{W}\right)$ increases to $\infty$ with $r$. This condition, however, is more restrictive. In the setting of Theorem 5.1, $\operatorname{Vol}\left(D_{r}\right)=\operatorname{Vol}\left(B_{T(r)}^{W}\right) \leq \operatorname{Vol}\left(B_{s(r)}^{W}\right)$ for all $r$, so we have inequality (4.6), and the existence of $T(R)$ is guaranteed without any additional hypothesis on the volume of the model space.

\section{INTRINSIC VERSIONS}

In this section we consider the intrinsic versions of Theorems 5.1 and 5.3 assuming that $P^{m}=N^{n}$. In this case, the extrinsic distance to the pole $p$ becomes the intrinsic distance in $N$, so, for all $r$ the extrinsic domains $D_{r}$ become the geodesic balls $B_{r}^{N}$ of the ambient manifold $N$. Then, for all $x \in P$

$$
\begin{aligned}
\nabla^{P} r(x) & =\nabla^{N} r(x), \\
H_{P}(x) & =0 .
\end{aligned}
$$

As a consequence, $\left\|\nabla^{P} r\right\|=1$, so $g(r(x))=1$ and $\mathcal{C}(x)=h(r(x))=0$, the stretching function becomes the identity $s(r)=r, W(s(r))=w(r)$, and the isoperimetric comparison space $C_{w, g, h}^{m}$ is reduced to the auxiliary model 
space $M_{w}^{m}$.

For this intrinsic viewpoint, we have the following isoperimetric and volume comparison inequalities.

Proposition 6.1 ([MP5]). Let $N^{n}$ denote a complete Riemannian manifold with a pole $p$. Suppose that the p-radial sectional curvatures of $N^{n}$ are bounded from below by the $p_{w}$-radial sectional curvatures of a $w$-model space $M_{w}^{n}$. Then, for all $R>0$

$$
\frac{\operatorname{Vol}\left(\partial B_{R}^{N}\right)}{\operatorname{Vol}\left(B_{R}^{N}\right)} \leq \frac{\operatorname{Vol}\left(\partial B_{R}^{w}\right)}{\operatorname{Vol}\left(B_{R}^{w}\right)}
$$

Furthermore,

$$
\operatorname{Vol}\left(B_{R}^{N}\right) \leq \operatorname{Vol}\left(B_{R}^{w}\right) .
$$

Theorem 6.2. Let $B_{R}^{N}$ be a geodesic ball of a complete Riemannian manifold $N^{n}$ with a pole $p$ and suppose that the $p$-radial sectional curvatures of $N^{n}$ are bounded from below by the $p_{w}$-radial sectional curvatures of a $w$-model space $M_{w}^{n}$. Assume that $M_{w}^{n}$ is balanced from above. Then

$$
\mathcal{A}_{1}\left(B_{R}^{N}\right) \geq \mathcal{A}_{1}\left(B_{T(R)}^{w}\right),
$$

where $B_{T(R)}^{w}$ is the Schwarz symmetrization of $B_{R}^{N}$ in the $w$-space $M_{w}^{n}$, i.e., it is the geodesic ball in $M_{w}^{n}$ such that $\operatorname{Vol}\left(B_{R}^{N}\right)=\operatorname{Vol}\left(B_{T(R)}^{w}\right)$.

Equality in (6.2) for some fixed radius $R$ implies that $T(R)=R$ and that $B_{R}^{N}$ and $B_{R}^{w}$ are isometric.

Proof. The proof follows the ideas of Theorem 5.1. In this case, since, $g(r)=$ 1 and $h(r)=0$, the second order differential operator $L$ agrees with the Laplacian on functions of one variable defined on the model spaces $M_{w}^{n}$,

$$
L f(r)=f^{\prime \prime}(r)+(n-1) \eta_{w}(r) f^{\prime}(r) .
$$

Solving the corresponding problem (3.8) on $[0, R]$ under this conditions, transplanting the solution $\psi(r)$ to the geodesic ball $B_{R}^{N}$, and applying Laplacian comparison analysis, (namely, using inequality (3.2) in Theorem 3.1 when $\psi^{\prime} \leq 0$ ), we obtain the inequality

$$
\Delta^{N} \psi(r(x)) \geq-1=\Delta^{N} E_{R}(x) .
$$

Since $\left\|\nabla^{P} r\right\|=1$, the sign of $\psi^{\prime \prime}(r)-\psi^{\prime}(r) \eta_{w}(r)$ is obsolete in this setting and we do not need to assume that $M_{w}^{n}$ is $w$-balanced from below.

Therefore, since $\psi(R)=E_{R}(R)=0$, the Maximum Principle, gives

$$
E_{R}(x) \geq \psi(r(x)) \text { for all } x \in B_{R}^{N},
$$


and we have

$$
\mathcal{A}_{1}\left(B_{R}^{N}\right)=\int_{B_{R}^{N}} E_{R} d \sigma \geq \int_{B_{R}^{N}} \psi d \sigma=\int_{B_{T(R)}^{w}} \psi^{*} d \tilde{\sigma},
$$

where $B_{T(R)}^{w}$ is the Schwarz symmetrization of the geodesic ball $B_{R}^{N}$ in the $w$-model space $M_{w}^{n}$, that is, the geodesic ball satisfying that $\operatorname{Vol}\left(B_{R}^{N}\right)=$ $\operatorname{Vol}\left(B_{T(R)}^{w}\right)$. From $(6.1)$, we know that $T(R) \leq R$.

Now, we consider the radial solution $E_{T(R)}^{w}(r)$ of the problem

$$
\begin{aligned}
\Delta^{M_{w}^{n}} E & =-1 \quad \text { on } B_{T(R)}^{w}, \\
\left.E\right|_{\partial B_{T(R)}^{w}} & =0 .
\end{aligned}
$$

With an argument analogous to that of Theorem 5.1, we conclude that

$$
\psi^{*}(t) \geq E_{T(R)}^{w}(t) \text { for all } t \in[0, T(R)],
$$

and then

$$
\mathcal{A}_{1}\left(B_{R}^{N}\right) \geq \int_{B_{T(R)}^{w}} \psi^{*} d \tilde{\sigma} \geq \int_{B_{T(R)}^{w}} E_{T(R)}^{w} d \tilde{\sigma}=\mathcal{A}_{1}\left(B_{T(R)}^{w}\right) .
$$

To prove the equality assertion, we must take into account that equality in (6.2) for some fixed radius $R>0$ implies equality in (6.4) for all $x \in B_{R}^{N}$. Then, the exponential map from the pole $p$ generates an isometry from $B_{R}^{N}$ onto $B_{R}^{w}$ in the way described in [MP4].

The following intrinsic version of Theorem 5.3 was stated and proved in [MP4].

Theorem 6.3. Let $B_{R}^{N}$ be a geodesic ball of a complete Riemannian manifold $N^{n}$ with a pole $p$ and suppose that the p-radial sectional curvatures of $N^{n}$ are bounded from above by the $p_{w}$-radial sectional curvatures of a $w$-model space $M_{w}^{n}$. Assume that $M_{w}^{n}$ is totally balanced. Then

$$
\mathcal{A}_{1}\left(B_{R}^{N}\right) \leq \mathcal{A}_{1}\left(B_{T(R)}^{w}\right),
$$

where $B_{T(R)}^{w}$ is the Schwarz symmetrization of $B_{R}^{N}$ in the $w$-space $M_{w}^{n}$, i.e., it is the geodesic ball in $M_{w}^{n}$ such that $\operatorname{Vol}\left(B_{R}^{N}\right)=\operatorname{Vol}\left(B_{T(R)}^{w}\right)$.

Equality in (6.5) for some fixed radius $R$ implies that $T(R)=R$ and that $B_{R}^{N}$ and $B_{R}^{w}$ are isometric.

Proof. We solve (3.8) under the same conditions as in the proof of Theorem 6.2 , and transplant the solution to the geodesic ball $B_{R}^{N}$. In this case, the $p$-radial sectional curvatures of $N$ are bounded from above by the $p_{w}$-radial sectional curvatures in $M_{w}^{n}$, and $\psi^{\prime}(r) \leq 0$ so we have the inequality

$$
\Delta^{N} \psi(r(x)) \leq-1=\Delta^{N} E_{R}(x) .
$$


Hence, $E_{R} \leq \psi$ on $B_{R}^{N}$ and we have inequality (5.1) using the same arguments as in the proof of Theorem 6.2.

The equality assertion follows from same considerations than in Theorem 6.2 .

Remark 1. Although we do not need the condition that the $w$-model space be balanced from below to conclude that $E_{R} \leq \psi$ on $B_{R}^{N}$, we need to guarantee that there is enough room for the symmetrization construction. In this setting, $\operatorname{Vol}\left(B_{R}^{N}\right) \geq \operatorname{Vol}\left(B_{R}^{w}\right)$ for each $R$, and the volume of the $w$-model may be finite. However, if the $w$-model space is $w$-balanced from below, $w^{\prime}(r)>0$ and the volume $\operatorname{Vol}\left(B_{r}^{w}\right)$ increases to $\infty$. For this reason, we assume that $M_{w}^{n}$ is totally balanced in Theorem 6.3 .

\section{Average mean exit time function}

The geometric average mean exit time from the extrinsic balls $D_{R}$, defined by the quotient $\mathcal{A}_{1}\left(D_{R}\right) / \operatorname{Vol}\left(D_{R}\right)$, was introduced in [MP4], with the purpose to give some idea about the volume-relative swiftness of the Brownian motion defined on the submanifold $P$ at infinity, in connection with the more classical properties like transience and recurrence.

As alluded to in the Introduction, we have been inspired partially by the works $[\mathrm{BBC}]$ and $[\mathrm{BG}]$, where the authors find upper bounds for the torsional rigidity of domains in Euclidean spaces which satisfy Hardy inequalities. These inequalities guarantee that the boundaries of the domains are not too thin so that the Brownian diffusion is guaranteed sufficient room for escape.

In our present setting, the thickness of the boundary is replaced by the isoperimetric inequalities (3.5) and (3.6), satisfied by our extrinsic domains in different curvature contexts, which controls whether the Brownian diffusion process is slow or fast at infinity.

For example, although Brownian diffusion is known to be transient in Euclidean spaces of dimensions larger than 2, it is not sufficiently swift, however, to give even a finite average of the mean exit time at infinity for geodesic balls, (see [MP4, Corollary 5.2]). Concerning this observation, we gave in [MP4] a set of curvature restrictions which give finiteness of the average mean exit time at infinity for minimal submanifolds. We shall present in Corollary 7.3, a generalization of this result for submanifolds with controlled radial mean curvature. On the other hand, in Corollary 7.2 a dual version of this result is presented in the sense that we find a set of curvature bounds which guarantee that the average of the mean exit time at infinity 
is infinite, (thus obtaining a set of curvature restrictions under which the Brownian diffusion process defined on the submanifold is slow).

In the following results, we shall denote as $\overline{\mathbb{R}}$ the extended real line so that $\overline{\mathbb{R}}_{+}=\mathbb{R}_{+} \cup\{\infty\}$.

Proposition 7.1. Let $M_{w}^{m}$ be a w-model space with infinite volume. Let us suppose that the following limit exists:

$$
\lim _{R \rightarrow \infty} q_{W}(R)=q_{W}(\infty) \in \overline{\mathbb{R}}_{+} .
$$

Then the average mean exit time from the $R$-balls in these model spaces satisfies:

$$
\lim _{R \rightarrow \infty} \frac{\mathcal{A}_{1}\left(B_{R}^{w}\right)}{\operatorname{Vol}\left(B_{R}^{w}\right)}=q_{W}^{2}(\infty) \in \overline{\mathbb{R}}_{+}
$$

Proof. We apply L'Hospital's Rule to the differentiable functions in ]0, $\infty[$, $f(R)=\mathcal{A}_{1}\left(B_{R}^{w}\right)$ and $g(R)=\operatorname{Vol}\left(B_{R}^{w}\right)$. Using the fact that, in the model spaces, the derivative of the volume of the geodesic balls is equal to the volume of the geodesic spheres, see (2.7), and equation (2.10) we have

$$
\lim _{R \rightarrow \infty} \frac{\mathcal{A}_{1}\left(B_{R}^{w}\right)}{\operatorname{Vol}\left(B_{R}^{w}\right)}=\lim _{R \rightarrow \infty} q_{W}^{2}(R)=\left(\lim _{R \rightarrow \infty} q_{W}(R)\right)^{2}
$$

Remark m. If $q_{W}^{2}(\infty)>0$, then, since the volume of the space is infinite, $\lim _{R \rightarrow \infty} \operatorname{Vol}\left(B_{R}^{w}\right)=\infty$ and from inequality $(7.2), \lim _{R \rightarrow \infty} \mathcal{A}_{1}\left(B_{R}^{w}\right)=\infty$.

As corollaries of Proposition 7.1 and Theorems 5.1 and 5.3, we have the following results:

Corollary 7.2. Let $\left\{N^{n}, P^{m}, C_{w, g, h}^{m}\right\}$ denote a comparison constellation bounded from below. Assume that $M_{W}^{m}=C_{w, q, h}^{m}$ is w-balanced from below, that it is $W$-balanced from above, and that it has infinite volume. Let $D_{R}$ be an extrinsic $R$-ball in $P^{m}$, with center at a point $p \in P$ which also serves as a pole in $N$. If the volume of the submanifold $P$ is infinite, and $\lim _{R \rightarrow \infty} q_{W}(R)=q_{W}(\infty)=\infty$ then

$$
\lim _{R \rightarrow \infty} \frac{\mathcal{A}_{1}\left(D_{R}\right)}{\operatorname{Vol}\left(D_{R}\right)} \geq q_{W}^{2}(\infty)=\infty
$$

Proof. Applying Theorem 5.1,

$$
\lim _{R \rightarrow \infty} \frac{\mathcal{A}_{1}\left(D_{R}\right)}{\operatorname{Vol}\left(D_{R}\right)} \geq \lim _{R \rightarrow \infty} \frac{\mathcal{A}_{1}\left(B_{T(R)}^{W}\right)}{\operatorname{Vol}\left(B_{T(R)}^{W}\right)},
$$

where $B_{T(R)}^{W}$ is the Schwarz symmetrization of $D_{R}$ in the model space $M_{W}^{m}$. 
Now, suppose that $\lim _{R \rightarrow \infty} T(R)=T_{\infty}<\infty$. Then,

$$
\operatorname{Vol}(P)=\lim _{R \rightarrow \infty} \operatorname{Vol}\left(D_{R}\right)=\lim _{R \rightarrow \infty} \operatorname{Vol}\left(B_{T(R)}^{W}\right)=\operatorname{Vol}\left(B_{T_{\infty}}^{W}\right)<\infty,
$$

which leads to a contradiction. As a consequence, $T(R)$ goes to $\infty$ and we can replace $T(R)$ by $R$ in the limit construction in the model space, that is

$$
\lim _{R \rightarrow \infty} \frac{\mathcal{A}_{1}\left(D_{R}\right)}{\operatorname{Vol}\left(D_{R}\right)} \geq \lim _{R \rightarrow \infty} \frac{\mathcal{A}_{1}\left(B_{R}^{W}\right)}{\operatorname{Vol}\left(B_{R}^{W}\right)} .
$$

The result follows now applying Proposition 7.1. To do that, we must check that

$$
\lim _{R \rightarrow \infty} \operatorname{Vol}\left(B_{R}^{W}\right)=\infty,
$$

but this follows from $\operatorname{Vol}\left(M_{W}^{m}\right)=\infty$. On the other hand, we assume that $\lim _{R \rightarrow \infty} q_{W}(R)=\infty \in \overline{\mathbb{R}}$.

Corollary 7.3. (see [MP4, Corollary 2.3]) Let $\left\{N^{n}, P^{m}, C_{w, 1, h}^{m}\right\}$ denote a comparison constellation bounded from above. Assume that $M_{W}^{m}=C_{w, 1, h}^{m}$ is $w$-balanced from below, is $W$-balanced from above, and has infinite volume. Let $D_{R}$ be an extrinsic $R$-ball in $P^{m}$, with center at a point $p \in P$ which also serves as a pole in $N$. Suppose that the model space geodesic spheres do not have 0 as a limit for their mean curvatures $\eta_{W}(R)$ as $R \rightarrow \infty$ and that these mean curvatures satisfy $\eta_{W}(r)>0$ for all $r>0$.

Then $\lim _{R \rightarrow \infty} q_{W}(R)=q_{W}(\infty)<\infty$ and

$$
\lim _{R \rightarrow \infty} \frac{\mathcal{A}_{1}\left(D_{R}\right)}{\operatorname{Vol}\left(D_{R}\right)} \leq q_{W}^{2}(\infty)<\infty
$$

Proof. Proceeding as in Corollary 7.2, and applying Theorem 5.3, we have firstly

$$
\lim _{R \rightarrow \infty} \frac{\mathcal{A}_{1}\left(D_{R}\right)}{\operatorname{Vol}\left(D_{R}\right)} \leq \lim _{R \rightarrow \infty} \frac{\mathcal{A}_{1}\left(B_{T(R)}^{W}\right)}{\operatorname{Vol}\left(B_{T(R)}^{W}\right)},
$$

where $B_{T(R)}^{W}$ is the Schwarz-symmetrization of $D_{R}$ in the model space $M_{W}^{m}$.

As in the proof of Corollary 7.2, we can replace $T(R)$ by $R$ in the limit construction in the model space, that is

$$
\lim _{R \rightarrow \infty} \frac{\mathcal{A}_{1}\left(D_{R}\right)}{\operatorname{Vol}\left(D_{R}\right)} \leq \lim _{R \rightarrow \infty} \frac{\mathcal{A}_{1}\left(B_{R}^{W}\right)}{\operatorname{Vol}\left(B_{R}^{W}\right)}=\lim _{R \rightarrow \infty} q_{W}(R),
$$

and we apply Proposition 7.1 , because by hypothesis, $\operatorname{Vol}\left(M_{W}^{m}\right)=\infty$, and, on the other hand, the limit

$$
\lim _{R \rightarrow \infty} q_{W}(R)<\infty .
$$


To see inequality (7.11) we use the fact that $\lim _{R \rightarrow \infty} \eta_{W}(R) \neq 0$. Then, as $q_{W}^{\prime}(R) \geq 0$, we have that $q_{W}(R) \eta_{W}(R) \leq \frac{1}{m-1}$, see [MP4, Observation 3.8], so, as $\eta_{W}(R) \geq 0$ for all $R$, we get

$$
q_{W}(R) \leq \frac{1}{(m-1) \eta_{W}(R)}
$$

Remark n. When $P$ is minimal, we may use $h=0$ as a bound for the $p$-radial mean curvature, and hence, since by hypothesis $g=1$, we have: $W=w$. In this case and by virtue of the balance conditions, the model space $M_{w}^{m}$ is totally balanced and then we have $\eta_{w}(R)>0$ for all $R>0$. Therefore, Corollary 7.3 clearly generalizes [MP4, Corollary 2.3 ].

\section{REFERENCES}

[Ba] C. Bandle, Isoperimetric Inequalities and Applications, Pitman Publishing Inc. (1980).

[BBC] R. Bañuelos, M. van den Berg, and T. Carroll, Torsional rigidity and expected lifetime of Brownian motion, J. London Math. Soc.(2) 66 (2002), 499-512.

[BG] M. van den Berg and P. B. Gilkey, Heat content and Hardy inequality for complete Riemannian manifolds, Bull. Lond. Math. Soc. 36 (2004), 577-586.

[Ch1] I. Chavel, Eigenvalues in Riemannian Geometry, Academic Press (1984).

[Ch2] I. Chavel, Isoperimetric inequalities. Differential geometric and analytic perspectives, Cambridge Tracts in Mathematics, 145. Cambridge University Press (2001).

[CLY] S. Y. Cheng, P. Li, and S. T. Yau, Heat equations on minimal submanifolds and their applications, Amer. J. Math. 106 (1984), 1033-1065.

[DCW] M. P. DoCarmo and F. W. Warner, Rigidity and convexity of hypersurfaces in spheres, J. Differential Geometry 4 (1970), 133-144.

[Dy] E. B. Dynkin Markov processes, Springer Verlag (1965).

[GreW] R. Greene and H. Wu, Function theory on manifolds which possess a pole, Lecture Notes in Math., vol. 699, Springer-Verlag, Berlin and New York (1979).

[Gri] A. Grigor'yan, Analytic and geometric background of recurrence and nonexplosion of the Brownian motion on Riemannian manifolds, Bull. Amer. Math. Soc. 36 (1999), 135-249.

[H] R. Z. Has'minskii, Probabilistic representation of the solution of some differential equations, in Proc. 6th All Union Conf. on Theor. Probability and Math. Statist. (Vilnius 1960), (1960)

[JK] L. P. Jorge and D. Koutroufiotis, An estimate for the curvature of bounded submanifolds, Amer. J. Math. 103 (1981), 711-725.

[Ma1] S. Markvorsen, On the mean exit time from a minimal submanifold, J. Diff. Geom. 29 (1989), 1-8 .

[Ma2] S. Markvorsen, On the heat kernel comparison theorems for minimal submanifolds, Proc. Amer. Math. Soc. 97 (1986), 479-482.

[MM] S. Markvorsen and M. Min-Oo, Global Riemannian Geometry: Curvature and Topology, Advanced Courses in Mathematics CRM Barcelona, Birkhäuser, Berlin (2003). 
[MP1] S. Markvorsen and V. Palmer, Generalized isoperimetric inequalities for extrinsic balls in minimal submanifolds, J. reine angew. Math. 551 (2002), 101-121.

[MP2] S. Markvorsen and V. Palmer, Transience and capacity of minimal submanifolds, GAFA, Geom. Funct. Anal. 13 (2003), 915-933.

[MP3] S. Markvorsen and V. Palmer, How to obtain transience from bounded radial mean curvature, Trans. Amer. Math. Soc. 357 (2005), 3459-3479.

[MP4] S. Markvorsen and V. Palmer, Torsional rigidity of minimal submanifolds, Proc. London Math. Soc. 93 (2006), 253-272.

[MP5] S. Markvorsen and V. Palmer, Extrinsic isoperimetric analysis on submanifolds with curvatures bounded from below, Preprint 2007, 39 pages.

[Mc] P. McDonald, Isoperimetric conditions, Poisson problems, and diffusions in Riemannian manifolds, Potential Analysis 16, (2002), 115-138.

[O'N] B. O'Neill, Semi-Riemannian Geometry; With Applications to Relativity, Academic Press (1983).

[Pa1] V. Palmer, Mean exit time from convex hypersurfaces, Proc. Amer. Math. Soc. 126 (1998), 2089-2094.

[Pa2] V. Palmer, Isoperimetric inequalities for extrinsic balls in minimal submanifolds and their applications, Jour. London Math. Soc. 60 (2) (1999), 607-616.

[PS] G. Pólya and G. Szegö, Isoperimetric Inequalities in Mathematical Physics, Princeton University Press (1951).

[Po] G. Pólya, Torsional rigidity, principal frequency, electrostatic capacity and symmetrization, Quart. Appl. Math. 6 (1948), 267-277.

[S] T. Sakai, Riemannian Geometry, Translations of Mathematical Monographs Volume 149, American Mathematical Society, 1996.

[Sp] M. Spivak, A comprehensive introduction to Differential Geometry, Publish or Perish Inc., Houston, (1979).

Departament de Matemàtiques, Universitat Jaume I, E-12071 Castelló, SPAIN.

E-mail address: ahurtado@mat.uji.es

Department of Mathematics, Technical University of Denmark, DK-2800 KGS. LyNGBY, DENMARK

E-mail address: S.Markvorsen@mat.dtu.dk

Departament de Matemàtiques, Universitat Jaume I, Castelló, Spain.

E-mail address: palmer@mat.uji.es 